LIN WANG, Ph.D.1,2

E-mail: jiangx@smm.neu.edu.cn

HONG WANG, Ph.D. ${ }^{1}$

(Corresponding author)

E-mail: 80090228@qq.com

XIN JIANG, Ph.D. ${ }^{1}$

E-mail: 25158734@qq.com

${ }^{1}$ School of Mechanical Engineering and Automation

Northeastern University; Shenyang 110819, China

2 Department of Mechanical Engineering, Shenyang

Institute of Engineering, Shenyang 110136, China
Human - Transport Interaction

Original Scientific Paper

Submitted: 30 Aug. 2016

Accepted: 26 Apr. 2017

\title{
A NEW METHOD TO DETECT DRIVER FATIGUE BASED ON EMG AND ECG COLLECTED BY PORTABLE NON-CONTACT SENSORS
}

\begin{abstract}
Recently, detection and prediction on driver fatigue have become interest of research worldwide. In the present work, a new method is built to effectively evaluate driver fatigue based on electromyography (EMG) and electrocardiogram (ECG) collected by portable real-time and non-contact sensors. First, under the non-disturbance condition for driver's attention, mixed physiological signals (EMG, ECG and artefacts) are collected by non-contact sensors located in a cushion on the driver's seat. EMG and ECG are effectively separated by FastICA, and de-noised by empirical mode decomposition (EMD). Then, three physiological features, complexity of EMG, complexity of ECG, and sample entropy (SampEn) of ECG, are extracted and analysed. Principal components are obtained by principal components analysis (PCA) and are used as independent variables. Finally, a mathematical model of driver fatigue is built, and the accuracy of the model is up to $91 \%$. Moreover, based on the questionnaire, the calculation results of model are consistent with real fatigue felt by the participants. Therefore, this model can effectively detect driver fatigue.
\end{abstract}

\section{KEYWORDS}

driver fatigue; electromyography; electrocardiogram; complexity; sample entropy;

\section{INTRODUCTION}

Driver fatigue is one of the top causes of car accidents, since the fatigued drivers are unable to adequately perceive, react and respond to situations on the road. However, driver fatigue is difficult to quantify, because there is no drowsiness test comparable to blood alcohol testing for drunk drivers. Therefore, it is clear that the detection and prevention of driver fatigue on the road are more and more important to avoid traffic accidents, and the study on driver fatigue has become interest of research worldwide [1].
Currently, physiological signals have become effective methods to evaluate driver fatigue because a person usually has little control over them, which makes them a reliable source of drivers' information. Probably, the physiological-based methods are the most logical methods. The physiological signals include electromyography (EMG), electrocardiogram (ECG), electroencephalogram (EEG), electro-oculogram (EOG), and respiration signals (RESP). Then, some physiological features are extracted from the signals, such as complexity, approximate entropy (ApEn), sample entropy (SampEn), heart rate variability (HRV), medium frequency (MF), integrated electromyography (IEMG), etc. These features have some relations with driver fatigue in the driving process. Lemke [2] showed that monitoring physiological signals both on-road and on a simulator indicated that this was a promising method for monitoring driver fatigue. Fu [3] simultaneously recorded EMG, EEG, RESP signals by wearable sensors and sent them to a computer by Bluetooth. Then, a dynamic fatigue detection model based on Hidden Markov Model (HMM) was proposed, and a novel way to detect driver fatigue was provided. Also, she thought ApEn of EMG had better relationship with driver fatigue. Lal and Craig [4] thought that ECG was an effective physiological signal to evaluate driver fatigue, and they found a significant and large decrease in heart rate in the driving process. Recently, an algorithm for the identification of alpha spindles was described by Simon [5]. He thought EEG alpha spindle parameters increased both fatigue detection sensitivity and specificity as compared to EEG alpha-band power.

However, challenges still remain in the physiological-based methods so far. (1) Single physiological signal or single physiological feature cannot reflect all of the fatigue-related information. So, one physiological 
signal or feature is insufficient to effectively evaluate driver fatigue, and their combined application may be a better way to obtain accurate results. (2) It is difficult to collect the physiological signals in a non-contact way because usually electrodes and wire need to be in contact with the drivers directly. Then the drivers will be inevitably disturbed by the measuring electrodes.

Aimed at these two challenges, the combined application of physiological features collected by portable real-time and non-contact sensors is investigated in the present work. Also, it is easy to make a product of this method and it can be commercialized due to its non-disturbance on driver's attention in the driving process. Based on the reference [6] and our preliminary investigation, the complexity of EMG, complexity of ECG, and SampEn of ECG are selected as physiological features in this paper.

\section{EXPERIMENTS}

\subsection{Non-contact sensors}

In experiments, the portable real-time and non-contact sensors are inserted into a cushion on the driver's seat (Figure 1) [7], which cannot disturb the driver's attention in the driving process. Therefore, they are very suitable for long-term physiological signal acquisition. In the non-contact sensors, there are two pieces of conductive knit fabric with the size of $12 \mathrm{~cm} \times 22 \mathrm{~cm}$. They are made of silver-plated nylon, and they are highly conductive with a surface resistivity of $<1 \Omega /$ sq. These two pieces of conductive fabric are used as electrodes to collect the mixed signals of EMG and ECG in non-contact way when the thickness of cloth is less than $2 \mathrm{~mm}$. Then, the physiological signals with variable electric potential can be seen as $\mathrm{AC}$ power. Skin and conductive fabric were regarded as the two plates of parallel-plate capacitor. The driver's cloth is the insulating material between the skin and the conductive fabric. This kind of structure is consistent a) Simulated driving experiments

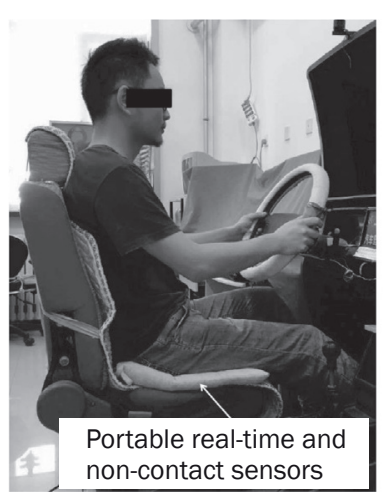

\section{Conductive Fabric}

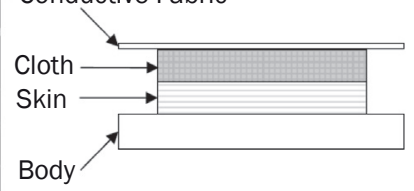

b) Sketch map of non-contact sensor
Figure 1 - Portable real-time and non-contact sensors are inserted into a cushion on the driver's seat with the capacitance coupling principle. The data acquisition system is Neuroscan 4.3, and the sampling frequency is $1,000 \mathrm{~Hz}$, the notch frequency is $50 \mathrm{~Hz}$. Data acquisition and data processing system in a computer are connected with the sensors in the cushion. As muscle contract, microvolt electric signal is created. Then, the mixed physiological signals (EMG, ECG and artefacts) of biceps femoris of each subject are collected and transferred to the data processing system.

\subsection{Participants}

NHTSA (the National Highway Traffic Safety Administration) has determined, based on the crash report evidence and drivers' self-report, that young people, particular males, between the age of 16 and 30 have the greatest risk for drowsy driving car crashes. So, 12 male and healthy volunteers (20-30 years old) were selected as participants in the driving experiments. All of the participants in the experiments were drivers who had more than two years of driving experience. They did not work night shifts, and they did not use prescription medication or had medical contraindications.

The participants were requested to drive continuously for $120 \mathrm{~min}$, and they were actively engaged on a driving simulator, such as turning the wheel, braking, accelerating, etc. The physiological signals were collected by the portable real-time and non-contact sensors in the cushion during the experiments. Also, during the driving experiments, the assistants help all the participants to fill in a questionnaire SOFI-25 form (Swedish Occupational Fatigue Inventory-25) at 10-minute intervals for the whole session. In order to minimize the fluctuation and individual differences. Many states were involved in the questionnaire, including energy, physical strength, comfort, and drowsiness for each participant. Then, the average of the above four items was considered as the score of one participant (Table 1). According to this form, we can roughly determine the alert state and fatigue state in the driving process based on the drivers' subjective feedback.

Table 1 - Subjective questionnaire about driver fatigue

\begin{tabular}{||c|l|c||}
\hline Items & \multicolumn{1}{|c|}{ States } & Score \\
\hline \hline 1 & $\begin{array}{l}\text { Energy: rapid heartbeat, lack of attention, } \\
\text { laziness, exhaustion of energy }\end{array}$ & $0 \sim 10$ \\
\hline 2 & $\begin{array}{l}\text { Physical strength: Paralysis, sweating, } \\
\text { physical exhaustion, out of spirits }\end{array}$ & $0 \sim 10$ \\
\hline 3 & $\begin{array}{l}\text { Comfort: Painful, breathing hard, passive, } \\
\text { boring }\end{array}$ & $0 \sim 10$ \\
\hline 4 & Drowsiness: Sleepy, yawning & $0 \sim 10$ \\
\hline & Average & $0 \sim 10$ \\
\hline
\end{tabular}




\subsection{Experimental design}

NHTSA has considered the following as factors that increase the risk of a car accident caused by driver fatigue. (1) Most fatigued-driver crashes occur between 4 a.m. - 6 a.m., midnight - 2 a.m., and mid-afternoon (2 p.m. - 4 p.m.). (2) Monotony of road scenario is also one of the factors for driver fatigue. So, in order to accelerate the participants becoming fatigued in the limited experimental time, the following parameters for driving simulation system are selected. Highway scene and automatic transmission car are selected, and the driving time is 2 p.m. - 4 p.m. The ambient temperature is $20^{\circ} \mathrm{C}$, and the humidity of air is $40 \sim 60 \%$ during the experiments.

\section{ANALYSIS METHODS}

\subsection{Data pre-processing}

\section{Original signals}

The original physiological signal collected from biceps femoris by the portable real-time and non-contact sensors is a mixed signal, including EMG, ECG, and artefacts. The noises are mainly the baseline noise induced by breath and drivers' body movement, the $50 \mathrm{~Hz}$ electronic noise, and other artificial contaminations. So, it can be defined as the sum of different components (Equation 1).

$X(t)=A\left[\begin{array}{l}S_{E M G}(t) \\ S_{E C G}(t)\end{array}\right]+\sum_{i=1}^{n} N_{i}(t)$

where: $A$ is an unknown mixing matrix which represents the weight of EMG signal $\left(S_{E M G}\right)$ and $E C G$ signal $\left(S_{E C G}\right)$. Ni represents various noises, and $t$ is a certain time during driving experiments. Figure 2 gives a typical 5-second original signal, and any useful physiological features cannot be found in the original signal. Therefore, the separation and de-noise for EMG and ECG from the original signals are the first work done

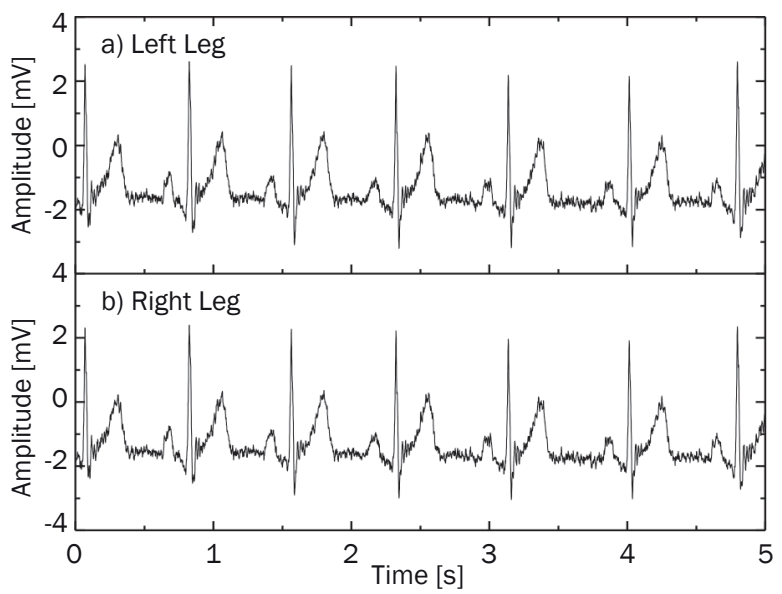

Figure 2 - Original mixed signal containing EMG, ECG, and various noises collected by non-contact sensors before the extraction and analysis of the physiological features.

\section{Separation of EMG and ECG}

FastICA is an efficient and popular algorithm for independent components analysis invented by Hyvärine[8]. Like most ICA algorithms, FastICA seeks an orthogonal rotation of data, through a fixedpoint iteration scheme that maximizes the measure of non-Gaussianity of the rotated components. Non-Gaussianity serves as a proxy for statistical independence, which is a very strong condition and requires infinite data to be verified. Therefore, FastICA is used to separate EMG and ECG from the original signal in the present work. Figure 3 gives a typical separated EMG and ECG from the original signals (Figure 2).

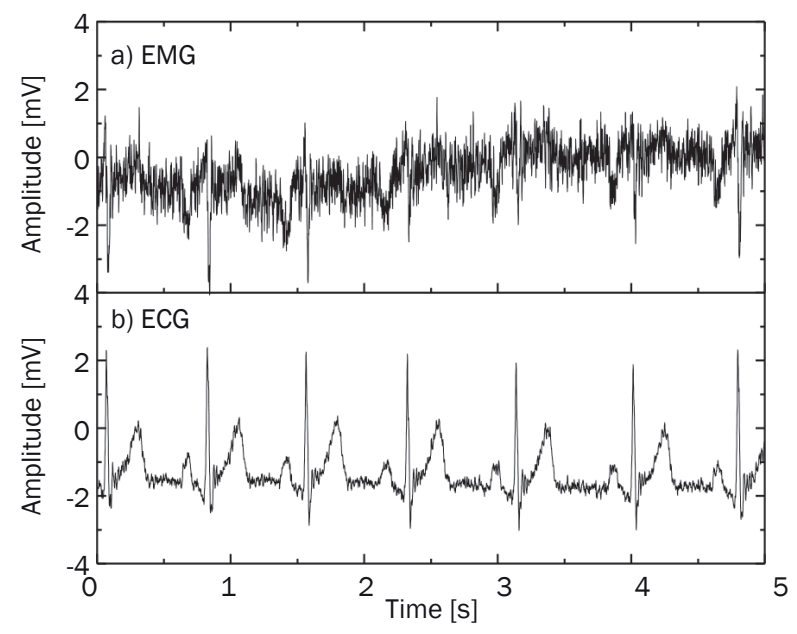

Figure 3 - Separated EMG and ECG from original signals

\section{De-noise for EMG and ECG}

EMG and ECG can be separated from original signal by FastICA, but there are still some noises and contamination in them. So, de-noise for them is necessary for further extraction of physiological features. Empirical Mode Decomposition (EMD) is a sifting process which is used to de-noise for EMG and ECG in the present work. With the help of EMD, a signal can be shifted layer by layer, and a series of IMFs (Intrinsic Mode Function) can be obtained. Then the frequency spectrum of these IMFs can be obtained by fast Fourier transform (FFT), mapping them in a frequency domain. In the frequency domain, the frequency of different IMFs is different. The earlier IMF sifted from the original signal has the higher frequency, the later IMF sifted from the signal has the lower frequency. EMG is reconstructed by the IMFs within $10-300 \mathrm{~Hz}$ [9], and other IMFs are deleted as noises. Similarly, ECG is reconstructed by the IMFs within $0-50 \mathrm{~Hz}[10$, 11]. Consequently, the noises of original signals can be effectively weakened by the reconstruction of these satisfied frequency spectra, and de-noised EMG and 
ECG can be obtained. For example, Figure 3 gives the 5-second separated EMG and ECG. The EMG and ECG are de-noised by EMD, and then they are free from noise and artificial contamination, which is shown in Figure 4.

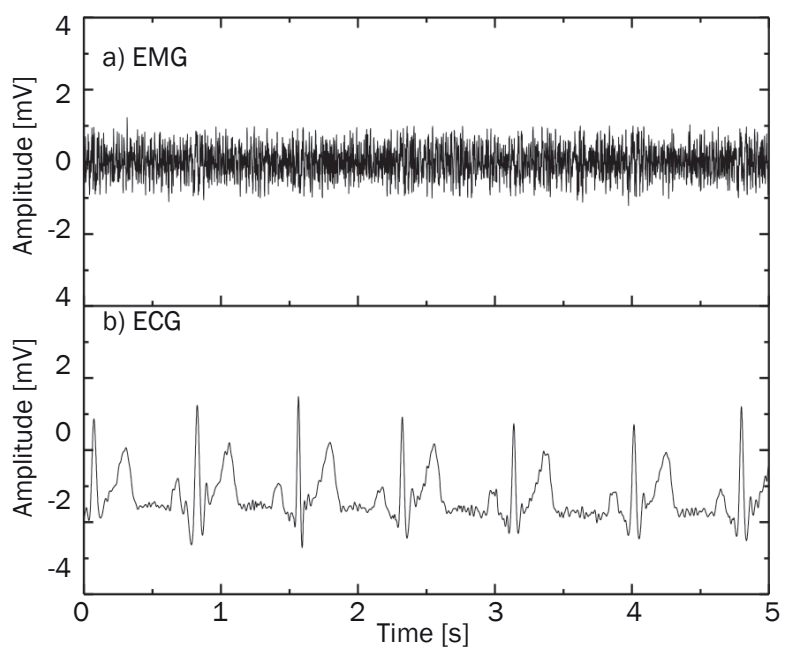

Figure 4 - De-noised EMG and ECG by EMD

\subsection{Features extraction}

\section{Lempel-Ziv complexity}

Lempel-Ziv complexity is a computational intensive method. It uses the relative information between two sequences. In the present work, EMG and ECG signals are needed to be converted into binary sequence. For EMG and ECG signals, first, the median value is calculated. Second, the signal data higher than the median is set as 1 , and the signal data lower than median is set as 0 . Then, these 0,1 points constitute a simplified sequence of EMG signals, represented as a binary sequence $P=[x(1), x(2), \ldots, x(n)]$. The Lempel-Ziv complexities of EMG and ECG are calculated based on the following algorithm [12, 13]:

1) For a given binary sequence $P=[x(1), x(2),, x(n)], S$ and $Q$ represent its subsequences. Initially, $S=x(1)$, $Q=x(2) . S Q$ denotes the concatenation of $S$ and $Q$, whereas the sequence $S Q v$ is derived from $S Q$ after its last character is deleted ( $v$ means the operation of deleting its last character in the sequence).

2) Judge whether $Q$ is a subsequence of $S Q v$. If $Q$ is a subsequence of $S Q v$, it means that the character of $Q$ can be copied from $S$. Then cascade the next character of $P$ to $Q$. If $Q$ is not a subsequence of $S Q v$, it means that the characters of $S Q v$ are inserted characters. Cascade $Q$ to $S, S=S Q$. Reconstruct $Q$. Then $Q$ is the next character of $P$.

3) Repeat 1 and 2, until the last character of $P$ is included in $Q$.

4) When $Q$ is cascade to $S$, a new pattern is obtained. The number of the new pattern is given by the calculation of complexity $c(n) ; c(n)$ is independent of the length of sequence. In order to calculate the complexity, it should be normalized.

5) Lempel and Ziv have proven that $c(n)$ tends to be a fixed value " $n / \log , n$ " as $n \rightarrow \infty$. For a binary sequence, $l=2$. Then, the normalized equation on the normalized Lempel-Ziv complexity is:

Complexity $=\frac{c(n) \log _{\jmath} n}{n}$

\section{Sample entropy}

Compared with ApEn, SampEn eliminates selfmatch and can speed up the model calculation [1416]. The calculation method of SampEn $(m, r, N)$ is listed as follows, where $m$ is the length of the sequences to be compared, $r$ is the tolerance for accepting matches, and $N$ is the length of the time series:

1) For a given time series of $N$ points (e.g. ECG), $x(1)$, $x(2) \ldots, x(n)$, forms $N-m+1$ vectors $X^{m}(i)$,

$$
\begin{aligned}
& X^{m}(i)=[x(i), x(i+1), \ldots, x(i+m-1)], \\
& 1 \leq i \leq 1+N-m
\end{aligned}
$$

2) The difference between two such vectors is defined as the maximum difference of their corresponding scalar components. It is,

$$
\begin{aligned}
& d\left[X^{m}(i), X^{m}(j)\right]=\max [|x(i+k)-x(j+k)|], \\
& 0 \leq k \leq m-1, \quad i \neq j, \quad 1 \leq j \leq N-m
\end{aligned}
$$

3) For a given $r, B_{i}^{m}(r)$ is the ratio of the number of $d\left[X^{m}(i), X^{m}(j)\right]<r$ to “N-m+1". It is,

$B_{i}^{m}(r)=\frac{\text { the number of } d\left[X^{m}(i), X^{m}(j)\right]<r}{N-m+1}$,

$i \neq j$

4) $B^{m}(r)$ is the average value of $B_{i}^{m}(r)$.

$$
B^{m}(r)=\frac{B_{1}^{m}+B_{2}^{m}+\ldots B_{N-M}^{m}}{N-M}
$$

5) $m$ is increased to $m+1$, repeat the $1^{\text {st }}$ step to $4^{\text {th }}$ step, and $B^{m+1}(r)$ is obtained.

6) Then, SampEn is given by the following equation.

$\operatorname{SampEn}(m, r, N)=-\operatorname{In}\left[\frac{B^{m+1}(r)}{B^{m}(r)}\right]$

By repeated testing, the SampEn of ECG has the best discrimination on driver fatigue when the parameters used in the present work are chosen as $m=2$ and $r=0.15 \times \mathrm{SD}$ (SD is the standard deviation of the original data) $[17,18]$.

\subsection{Model building}

In the present work, the detection model for driver fatigue is built by the combination of principal components analysis (PCA) and multiple linear regressions. 
Principal components analysis

Based on the calculation of section 3.2, the physiological features may be obtained. But usually, the features are correlated variables. The uncorrelated variables are necessary in multiple linear regressions. In order to obtain the uncorrelated variables and avoid pseudo regression, principal components analysis (PCA) is used to analyse the physiological features in the present work. The three features are replaced by the principal components, the useful information is kept, and the redundant information is eliminated. Then, the principal components are used as the independent variable matrix in the multiple regressions equation, which can ensure the validity and rationality of the driver fatigue model built in our work. Also, the dimensionality of features is reduced by PCA, which can speed up the model calculation. The algorithm on PCA can be found in reference $[19,20]$.

\section{Multiple linear regression}

Multiple linear regression is a statistic method to study the linear relation between one dependent variable and multiple independent variables. The calculation principle may be found in reference [21]. In the present work, the coefficients are calculated by the statistical and analytical software SPSS19.0.

\section{EXPERIMENTAL RESULTS}

The variations of physiological features trend during driving process are shown in Figure 5. In this figure, $30 \mathrm{~s}$ of physiological features are extracted approximately every 10 minutes. The first point represents the average value of one feature of all participants in about 9.5-10 minutes; the second point represents the average value of the feature of all participants in about 19.5-20 minutes, and so on. So, in total there are twelve points on each curve (total driving time is $120 \mathrm{~min}$ ). The recorded signals may contain the participants' movement-related EMG in addition to the claimed muscle tension. In order to solve this problem, if there are movement-related actions and the signal amplitude suddenly changes at the time of signal extraction, we can change the extraction time and select a time before or after the action. Therefore, the movement-related EMG may be avoided during the data analysis process.

From Figure 5, it can be concluded that the complexity of EMG, complexity of ECG, and SampEn of ECG decrease with the increase of the driving time, indicating that fatigue degree increases. After about 90minutes, the decreasing speed becomes slow, indicating deeper driver fatigue. Although the circumstance encountered in the driving process is different, (1) the regularity of downward trend is clear, and (2) the standard deviations of the features data are relatively small. Therefore, the complexity of EMG, complexity of
ECG, and SampEn of ECG have higher discrimination and stability in the judgment of driver fatigue.

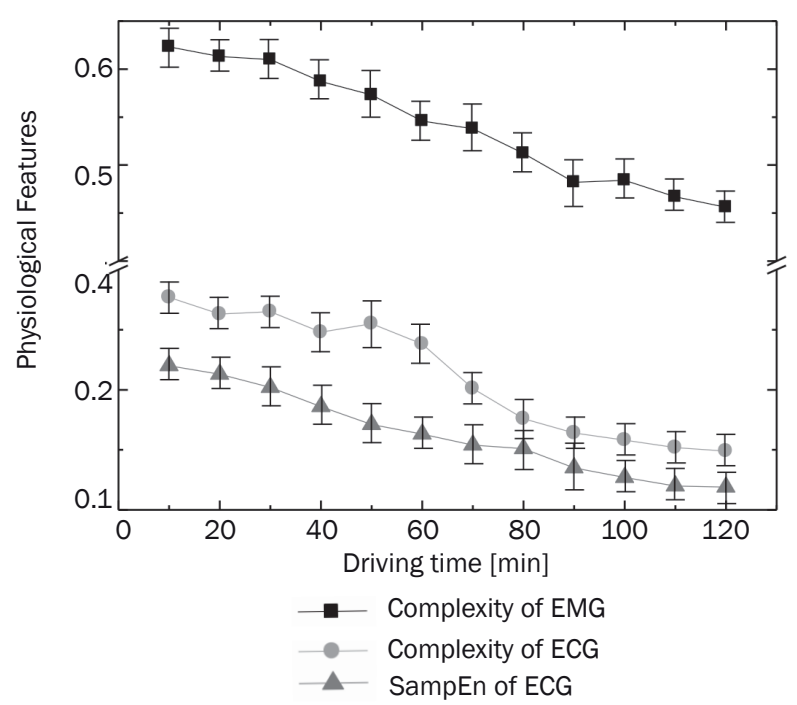

Figure 5 - Average physiological features of all participants

\section{MATHEMATICAL MODEL OF DRIVER FATIGUE}

\subsection{Three-D distribution of states}

In this paper, the results of subjective questionnaire SOFI-25 are used as labels for defining alert and fatigue state. Based on the questionnaire (Table 2), (1) basically all of the participants feel alert in 0-30 min, and the average score in 0-30 min is about 0.00-0.19; (2) they feel fatigue in $90-120 \mathrm{~min}$, and the average score in $90-120 \mathrm{~min}$ is about 5.27-6.29. So, there is significant difference in scores between alert state and fatigue state, and it can effectively evaluate the inter-subject variability. Therefore, 0-30 min of driving experiment is defined as alert state, and 90-120 min of driving experiment is defined as fatigue state. Also, this definition is similar as found in literature [22].

Time resolution and window length are crucial factors for the fatigue detection system and real-time driving. (a) In case of time resolution. In general, the feeling of driver fatigue is gradually generated, and it is an accumulation of a period of time. There is no significant change in a shorter time. But if the time interval is too long, the definition on driver fatigue will be not accurate enough. Therefore, a 5-minute interval is selected for calculating features. (b) In case of window length. The amount of data should be more than 1,000 when calculating the complexity and SampEn. Otherwise, the results will be affected by the amount of data, and will fail to be accurate [23]. Therefore, 30 -second signal is selected as the window length. 
Table 2 - Subjective scores of SOFI-25 questionnaire of participants (A-L) at different times

\begin{tabular}{||c|c|c|c|c|c|c|c|c|c|c|c|c|c||}
\hline \hline Time & $\mathrm{A}$ & $\mathrm{B}$ & $\mathrm{C}$ & $\mathrm{D}$ & $\mathrm{E}$ & $\mathrm{F}$ & $\mathrm{G}$ & $\mathrm{H}$ & $\mathrm{I}$ & $\mathrm{J}$ & $\mathrm{K}$ & $\mathrm{L}$ & Average \\
\hline \hline $0 \mathrm{~min}$ & 0.00 & 0.00 & 0.00 & 0.00 & 0.00 & 0.00 & 0.00 & 0.00 & 0.00 & 0.00 & 0.00 & 0.00 & 0.00 \\
\hline $10 \mathrm{~min}$ & 0.00 & 0.00 & 0.00 & 0.00 & 0.00 & 0.00 & 0.00 & 0.00 & 0.00 & 0.00 & 0.00 & 0.00 & 0.00 \\
\hline $20 \mathrm{~min}$ & 0.00 & 0.25 & 0.00 & 0.00 & 0.00 & 0.00 & 0.00 & 0.00 & 0.00 & 0.00 & 0.00 & 0.00 & 0.02 \\
\hline $30 \mathrm{~min}$ & 0.00 & 0.50 & 0.00 & 0.00 & 0.25 & 0.00 & 0.25 & 0.50 & 0.00 & 0.50 & 0.00 & 0.25 & 0.19 \\
\hline $40 \mathrm{~min}$ & 0.75 & 1.00 & 0.25 & 0.50 & 0.75 & 0.00 & 1.00 & 0.50 & 0.25 & 1.00 & 0.00 & 0.75 & 0.56 \\
\hline $50 \mathrm{~min}$ & 1.25 & 1.50 & 1.50 & 1.00 & 0.75 & 1.00 & 2.00 & 1.25 & 1.00 & 1.75 & 0.75 & 1.50 & 1.27 \\
\hline $60 \mathrm{~min}$ & 1.50 & 2.00 & 2.50 & 1.75 & 1.50 & 1.50 & 2.50 & 1.50 & 2.00 & 2.50 & 1.75 & 2.25 & 1.94 \\
\hline $70 \mathrm{~min}$ & 3.25 & 3.25 & 4.00 & 3.00 & 2.75 & 3.50 & 4.00 & 3.00 & 3.50 & 3.25 & 3.00 & 3.75 & 3.35 \\
\hline $80 \mathrm{~min}$ & 3.50 & 4.25 & 4.50 & 3.50 & 3.50 & 3.75 & 4.75 & 3.50 & 4.50 & 3.75 & 3.25 & 4.00 & 3.90 \\
\hline $90 \mathrm{~min}$ & 4.75 & 5.50 & 5.25 & 4.50 & 5.75 & 5.00 & 5.50 & 4.50 & 4.75 & 6.00 & 5.25 & 6.50 & 5.27 \\
\hline $100 \mathrm{~min}$ & 5.50 & 6.25 & 5.25 & 5.75 & 6.75 & 5.25 & 6.00 & 4.75 & 5.25 & 6.50 & 5.75 & 6.75 & 5.81 \\
\hline $110 \mathrm{~min}$ & 5.50 & 6.50 & 6.00 & 5.75 & 6.75 & 5.50 & 6.25 & 5.25 & 6.00 & 6.50 & 5.75 & 6.75 & 6.04 \\
\hline $120 \mathrm{~min}$ & 6.00 & 7.00 & 6.25 & 5.75 & 7.00 & 5.50 & 6.25 & 5.50 & 6.25 & 7.00 & 6.25 & 6.75 & 6.29 \\
\hline
\end{tabular}

Then, the physiological features are calculated at 5-minute interval for alert state (total $30 \mathrm{~min}$ ) and fatigue state (total $30 \mathrm{~min}$ ). For each calculation, 30 seconds of signals are involved, and there are three features: complexity of EMG, complexity of ECG, and SampEn of ECG. In order to eliminate the individual difference and absolute value difference, the features are normalized. Then, in the three-dimensional coordinate system (three features are the three coordinate axes), for one participant, there are 6 alert points and 6 fatigue points. In total, for 12 participants, there are 72 alert points and 72 fatigue points (Figure 6).

From Figure 6 one can conclude that, in case of alert state, the values of three physiological features are bigger and are mainly distributed in the upper right corner. In case of fatigue state, the values of three fea-

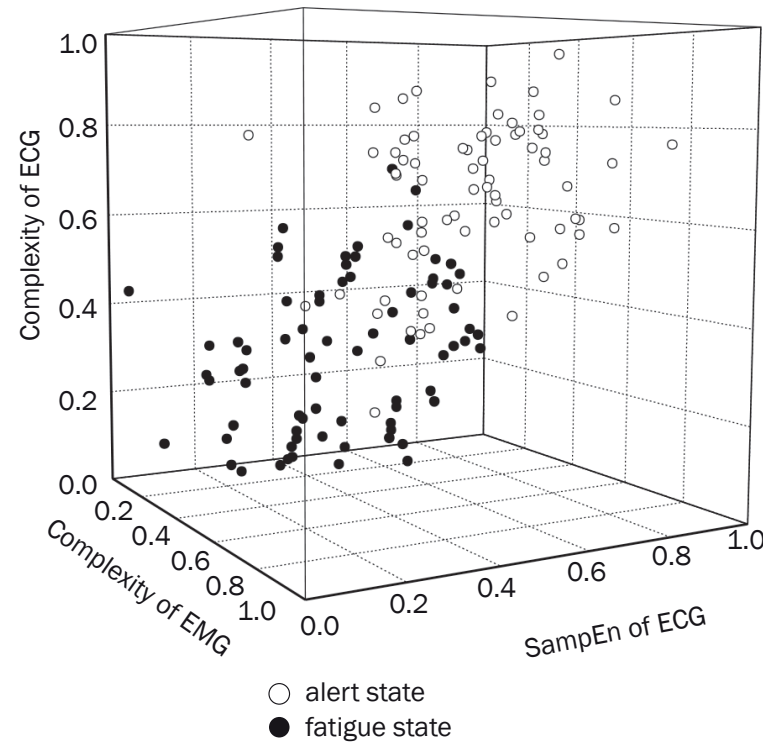

Figure 6 - Three-D distribution of alert state and fatigue state tures are smaller and mainly distributed in the lower left corner. But there is still some overlapping. This indicates that among the three features there is not only some useful and complementary information, but also some redundant information. The redundant information mainly comes from (1) the individual difference, (2) correlativity among the three features, and (3) different variation range of the three features. Therefore, if the three features are combined (replaced by some independent variables, keeping useful information and eliminating redundant information), the alert state and fatigue state may be better discriminated.

\subsection{Building driver fatigue model}

First, principal components analysis (PCA) is used to combine the complexity of EMG, complexity of ECG, and SampEn of ECG, and eliminate the redundant information among them. Three principal components $\left(C_{1}, C_{2}, C_{3}\right)$ are obtained by PCA, and they are uncorrelated variables. During the calculation, the principal components are given according to their variances. The variance represents the amount of useful data and the main variation trend of original data. So, we name it as contribution rate of each principal component. The contribution of principal components is shown in Figure 7. It can be seen that the contribution of the first principal component $C_{1}$ is the biggest one, the second one $C_{2}$ takes the second place, and the third one $C_{3}$ is the smallest. That means $C_{1}$ has most of the useful data and the main variation trend of original physiological features. The accumulative contribution of $C_{1}$ and $C_{2}$ is up to $92.01 \%$ (more than $90 \%$ ). But the contribution of $C_{3}$ is only $7.99 \%$. That means that most of the data in $C_{3}$ is redundant although probably it has little useful data. Therefore, by deleting $C_{3}$, although a small amount of useful data is lost, most of redundant data is removed, which can simplify and 
speed up the calculation. Then, $C_{1}$ and $C_{2}$ are selected as independent variables in the model, which are sufficient to describe the information on driver fatigue.

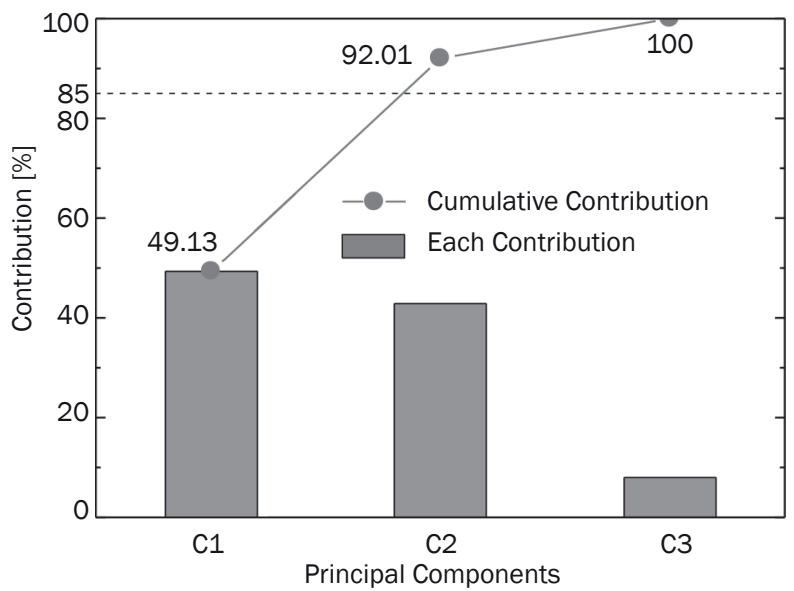

Figure 7- Contributions of principal components

Equation 8 gives the coefficient matrix of principal components, where $C_{1}, C_{2}, C_{3}$ represent $1^{\text {st }}, 2^{\text {nd }}, 3^{\text {rd }}$ principal component, respectively. $X_{1}$ represents the complexity of EMG, $X_{2}$ represents the complexity of $E C G$, and $X_{3}$ represents the SampEn of ECG. The coefficients represent the weight of each feature in a principal component. $C_{1}$ and $C_{2}$ are the linear combination of the three features. Then, useful information is kept, and the redundant information is eliminated. Moreover, the three dimensional physiological features $\left(X_{1}, X_{2}, X_{3}\right)$ are replaced by two dimensional principal components $\left(C_{1}, C_{2}\right)$, which can speed up the model calculation.

$$
\left[\begin{array}{l}
C_{1} \\
C_{2} \\
C_{3}
\end{array}\right]=\left[\begin{array}{ccc}
0.5082 & 0.6017 & 0.6243 \\
0.8675 & -0.3450 & -0.4609 \\
-0.5144 & 0.3913 & 0.3324
\end{array}\right] \cdot\left[\begin{array}{l}
X_{1} \\
X_{2} \\
X_{3}
\end{array}\right]
$$

Based on the above PCA, $C_{1}$ and $C_{2}$ are independent variables in the mathematical model. An integrated fatigue index $\Phi$ is proposed as the dependent variable. Then, according to the multiple linear regressions theory, the mathematical model of driver fatigue is given by Equation 9. In the equation, $\Phi>0$ denotes the alert state, and $\Phi<0$ denotes the fatigue state.

$\Phi=-1.509+1.806 \cdot C_{1}-0.577 \cdot C_{2}$

\subsection{Validity of the model}

In the present work, two validation methods (10fold cross validation and state validation) are used to verify the driver fatigue model. In case of 10 -fold cross validation, 144 points are divided into 10 groups. Any 9 groups take turns to be used as the training group, and 1 group is used as the test group. The results of 10-fold cross validation are listed in Table 3. It can be seen that the average calculation accuracy of the test group in the model is $91.04 \%$.

Table 3- Model validation by 10 -fold cross validation

\begin{tabular}{||c|c|c||}
\hline Test No. & $\begin{array}{c}\text { Accuracy of training } \\
\text { group }(\%)\end{array}$ & $\begin{array}{c}\text { Accuracy of test } \\
\text { group (\%) }\end{array}$ \\
\hline \hline $1^{\text {st }}$ test & 92.35 & 91.28 \\
\hline $2^{\text {nd }}$ test & 94.64 & 90.13 \\
\hline $3^{\text {rd }}$ test & 92.20 & 90.41 \\
\hline $4^{\text {th }}$ test & 88.95 & 90.82 \\
\hline $5^{\text {th }}$ test & 95.06 & 91.40 \\
\hline $6^{\text {th }}$ test & 91.44 & 93.21 \\
\hline $7^{\text {th }}$ test & 91.58 & 90.93 \\
\hline $8^{\text {th }}$ test & 95.64 & 92.71 \\
\hline $9^{\text {th }}$ test & 89.98 & 89.76 \\
\hline $10^{\text {th }}$ test & 93.84 & 89.78 \\
\hline Average & 92.57 & 91.04 \\
\hline
\end{tabular}

State validation results are listed in Table 4. It can be seen that the calculation accuracy of the model is $93.75 \%$. It is significantly higher than the traditional non-PCA model. In state validation, the alert state and the fatigue state are defined based on the participants' real feelings. So, the calculation results of the model are consistent with the real fatigue that the participants feel. Therefore, the mathematical model of driver fatigue built in this paper has higher accuracy based on the state validation and 10 -fold cross validation.

\section{DISCUSSION}

\subsection{Discussion on the model}

There are two key points for the effectiveness and rationality of the mathematical model of driver fatigue: (1) $C_{1}$ and $C_{2}$ shall have clear discrimination for alert state and fatigue state. (2) $C_{1}$ and $C_{2}$ shall be uncorrelated variables.

Table 4- Comparison between non-PCA model and PCA model

\begin{tabular}{|c|c|c|c|c|c|c|}
\hline \multirow{2}{*}{ Actual state } & \multicolumn{3}{|c|}{ State calculated by non-PCA model } & \multicolumn{3}{|c|}{ State calculated by PCA model } \\
\hline & Alert state & Fatigue state & Accuracy & Alert state & Fatigue state & Accuracy \\
\hline Alert state (72 points) & 61 points & 11 points & $84.72 \%$ & 67 points & 5 points & $93.06 \%$ \\
\hline Fatigue state (72 points) & 13 points & 59 points & $81.94 \%$ & 4 points & 68 points & $94.44 \%$ \\
\hline Total (144 points) & \multicolumn{2}{|c|}{ Average } & $83.33 \%$ & \multicolumn{2}{|c|}{ Average } & $93.75 \%$ \\
\hline
\end{tabular}




\section{Effectiveness of the model}

$C_{1}$ and $C_{2}$ should have clear discrimination. This is the key point for the effectiveness of the model. The $t$-test is used in this paper to verify their discrimination. Based on the calculation of t-test, the significance indices of $C_{1}$ and $C_{2}$ are 0.001 and 0.002 , respectively. Both of them are less than 0.05 , indicating that there is clear discrimination when $C_{1}$ and $C_{2}$ are used to discriminate alert state and fatigue state. Therefore, the principal components $C_{1}$ and $C_{2}$ may reveal the intrinsic regularity and may be the independent variables to increase the accuracy in evaluating driver fatigue.

\section{Rationality of the model}

$C_{1}$ and $C_{2}$ should be uncorrelated variables. This is the key point for the rationality of the model (calculated by multiple linear regressions). Basically, $C_{1}$ and $\mathrm{C}_{2}$ should be uncorrelated variables because they are the principal components calculated by PCA. In order to further verify it and avoid pseudo regression, the correlation between them is calculated by Pearson correlation coefficient algorithm (Equation 10) [24]. Based on the calculation of the equation, the correlation coefficient between $C_{1}$ and $C_{2}$ at any time is about $r=0.0$. Therefore, $C_{1}$ and $C_{2}$ are uncorrelated independent variables, which can ensure the rationality of the model.

$$
=\frac{r\left(C_{1}, C_{2}\right)=}{\sqrt{n \sum_{i=1}^{i=144} C_{i=1}^{i} C_{1 i}^{2}-\left(\sum_{i=1}^{i=144} C_{2 i}-\sum_{i=1}^{i}\right)^{2}} \sqrt{n \sum_{i=1}^{i=144} C_{2 i}-\left(\sum_{i=1}^{i=14} C_{2 i}\right)^{2}}}
$$

\section{Conditions on multiple regressions}

The main conditions for regression analysis are normality distribution of data and homoscedasticity. These conditions must be satisfied. Otherwise, the model will be meaningless. In the present work, the data are satisfied with normality distribution. There are two important factors for homoscedasticity: (1) large data noise, and (2) confounding variables. For case (1), EMD is used to de-noise for the separated EMG and ECG signals. Then, basically all of the noises have been effectively removed. For case (2), if there are colinearity and multicolinearity between independent variables, that means some redundant information exists in the signals. In the present work, PCA is used to remove the redundant information and keep useful information in the signals, and three principal components are obtained. Two of them $\left(C_{1}\right.$ and $C_{2}$, the total contribution is up to $92.01 \%$ ) are used in building the model. Also, at any time, the correlation between $C_{1}$ and $C_{2}$ is about 0.0 by Pearson correlation coefficient algorithm. So, $C_{1}$ and $C_{2}$ are uncorrelated independent variables. This means that the data are satisfied with homoscedasticity. Therefore, the accuracy of the model built in this paper is higher (up to $91 \%$ ) due to EMD and PCA.

\subsection{Expectation of the method}

Based on the above analysis, our work in this paper can effectively detect driver fatigue according to the following steps: (1) collect mixed EMG and ECG signals by portable real-time and non-contact sensors; (2) separate and de-noise EMG and ECG; (3) extract physiological features, including complexity of EMG, complexity of ECG, and SampEn of ECG; (4) obtain two principal components $\left(C_{1}\right.$ and $\left.C_{2}\right)$ by PCA; (5) calculate by our mathematical model of driver fatigue.

Also, this kind of system is easy to make product and be commercialized. The portable non-contact sensors are inserted into the cushion on the driver's seat, which cannot disturb the driver's attention in the driving process. The system of features extraction and model calculation can be connected with sensors in the cushion. When the fatigue state occurs calculated by the model, an alarm located in the car will automatically make a call. Then, the driver fatigue may be effectively detected. Calculation speed is one of the limitations for real application of this approach. In the present work, the model calculation may be significantly speeded up by using SampEn and PCA. This is important for online/real-time fatigue detection, and it is also an advantage of the model built in the present work.

It should be pointed out that the present work is carried out based on the experiments in the driving simulator. Some investigation on real driving (taxi drivers and highway drivers) will be done soon. This is one of important components for the effectiveness and rationality of the driver fatigue model. Currently, the driver fatigue detection system contains many steps, including window selection, normalization of features, application of PCA, building and calculation of the model etc. So actually, there is a lot of work to be done due to the complicated mechanism of driver fatigue. In the future, our main works will focus on the integration of data acquisition, calculation, analysis, and alarm. The related experimental results and conclusions will be published in the future. Consequently, a commercial product about detection and prevention of driver fatigue will be finally developed.

\section{CONCLUSION}

The main findings in this paper could be summarized as follows:

1) Under the non-disturbance condition for driver's attention in the driving process, mixed physiological signals (EMG, ECG and artefacts) are collected by portable real-time and non-contact sensors located in a cushion on the driver's seat. EMG and ECG can be effectively separated by FastICA, and de-noised by EMD. Then, three physiological features, complexity of EMG, complexity of ECG, and SampEn of ECG, are extracted and analysed. All of 
three features decrease during the 120 min driving process. After about 90 min, the decreasing speed becomes slow, indicating deeper driver fatigue.

2) Two principal components $\left(C_{1}\right.$ and $\left.C_{2}\right)$ are obtained by PCA, which can replace the three physiological features, keeping useful information and eliminating redundant information. Then, based on multiple linear regressions, an integrated fatigue index $\Phi$ is proposed, and a mathematical model of driver fatigue is built by using principal components as the independent variables. According to the10-fold cross validation and state validation, the calculation accuracy of the model is up to $91 \%$. Moreover, based on the questionnaire, the calculation results of the model are consistent with the real fatigue felt by the participants. Therefore, our work in this paper can effectively detect driver fatigue.

3) The method used in the present work is easy to make product and be commercialized due to its portable non-disturbance on driver's attention in the driving process. Also, by using SampEn and PCA, the model calculation is speeded up. This is important for the application of the model in actual driving process, and it is also an advantage of this model.

\section{ACKNOWLEDGMENT}

The authors wish to acknowledge the contributions of associates and colleagues in Northeastern University, China and Shenyang Institute of Engineering, China.

\section{王琳（博士） 1,2}

E-mail: jiangx@smm.neu.edu.cn

王宏（博士，通讯作者） 1

E-mail: 80090228@qq.com

姜金金 (博士) 1

E-mail: 25158734@qq.com

1 东北大学机械工程与自动化学院, 沈阳 110819 , 中国

2 沈阳工程学院机械学院, 沈阳, 110136, 中国

基于非接触传感器的肌电心电信号疲劳驾驶检测方 法

摘要

近年来, 疲劳驾驶的检测与预防引起了国内外 研究人员的关注。本文提出了一种基于非接触传感 器的肌电信号 (EMG) 和心电信号 (ECG) 疲劳驾驶 检测方法。首先, 在不影响驾驶员驾驶的情况下, 通过置于坐垫内的非接触传感器检测了驾驶员的混 合生理信号（包括EMG、ECG和噪声）。通过快速独 立分析FastICA对EMG和ECG进行粗分离, 通过经验 模态分解对粗分离后的EMG和ECG进行去燥。然后, 提取并分析了三种特征参数 (EMG复杂度、ECG复杂 度、ECG样本摘）。通过主成分分析PCA得到了两个 主成分, 并以此作为自变量, 建立了疲劳驾驶检测
的数学模型。该模型的正确率达 $91 \%$ 以上。而且, 基 于调查问卷，模型的计算结果与被试者的实际感觉 相一致。因此, 该模型可以有效地检测疲劳驾驶状 态

\section{关键词}

疲劳驾驶, 肌电信号, 心电信号, 复杂度, 样本熵

\section{REFERENCES}

[1] Santamaria J, Chiappa KH. The EEG of Drowsiness. New York: Demos Publications; 1987.

[2] Lemke M. Correlation between EEG and driver's actions during prolonged driving under monotonous conditions. Accident Analysis \& Prevention. 1982;14(1):7-17.

[3] Fu RR, Wang H, Zhao WB. Dynamic driver fatigue detection using hidden Markov model in real driving condition. Expert System with Application. 2016;63(C):397-411.

[4] Lal SKL, Craig A. Driver fatigue: electroencephalography and psychological assessment. Psychophysiology. 2002;39(3):313-321.

[5] Simon M, Schmidt EA, Kincses WE, Fritzsche M, Bruns A, Aufmuth C, Bogdan M, Rosenstiel W, Schrauf M. EEG alpha spindle measures as indicators of driver fatigue under real traffic conditions. Clinical Neurophysiology. 2011;122(6):1168-1178.

[6] Richman JS, Moorman JR. Physiological time-series analysis using approximate entropy and sample entropy. American Journal of Physiology Heart \& Circulatory Physiology. 2000;278(6):2039-2049.

[7] Fu RR, Wang $H$. Detection of driving fatigue by using noncontact EMG and ECG signals measurement system. International Journal of Neural System. 2014;24(3):1450006.

[8] Hyvärine A, Oja E. Independent component analysis algorithm and application. Neural Networks. 2000;13(4):411-430.

[9] Tscharner VV, Eskofier B, Federolf P. Removal of the electrocardiogram signal from surface EMG recordings using non-linearly scaled wavelets. Journal of Electromyography and Kinesiology. 2011;21(4):683-688.

[10] Poornachandra S, Kumaravel N. A novel method for the elimination of power line frequency in ECG signal using hyper shrinkage function. Digital Signal Processing. 2008;18(2):116-126.

[11] Sankari Z, Adeli H. Heart Saver: a mobile cardiac monitoring system for auto-detection of atrial fibrillation, myocardial infarction and atrio-ventricular block. Computers in Biology and Medicine. 2011;41(4):211-220.

[12] Lempel A, Ziv J. On the complexity of finite sequence. IEEE Transactions on Information Theory. 1976;22(1):75-81.

[13] Zhang $\mathrm{C}$, Wang $\mathrm{H}$, Wu MH. EEG-based expert system using complexity measures and probability density function control in alpha sub-band. Integrated Computer Aided Engineering. 2013;20(4):391-405.

[14] Pincus SM. Approximate entropy (ApEn) as a complexity measure. International Journal of Chaos. 1995;5(1):110-117.

[15] Grassberger P. Finite sample corrections to entropy and dimension estimates. Physics Letters A. 1988;128(6):369-373. 
[16] Foij O, Holcik J. Applying nonlinear dynamics to ECG signal processing. IEEE Engineering in Medicine and Biology. 1998;3(4):96-110.

[17] Zhang C, Wang H, Fu RR. Automated detection of driver fatigue based on entropy and complexity measures. IEEE Transactions on Intelligent Transportation System. 2014;15(1):168-177.

[18] Alcaraz R, Rieta JJ. A novel application of sample entropy to the electrocardiogram of atrial fibrillation. Nonlinear Analysis Real World Applications. 2010;11(2):1026-1035.

[19] Pearson K. On lines and planes of closest fit to systems of points in space. Philosophical Magazine. 1901;2(6):559-572.

[20] Fu RR, Wang H, Wang L. Detection of driver fatigue based on multi-physiological signals in wireless body area network. Journal of Northeastern University (Natural Science). 2014;35(6):850-853.

[21] Fu RR. Research of Driver Fatigue Recognition Based on Machine Learning [Ph.D. thesis, in Chinese]. Northeastern University, Shenyang, China; 2015. p. 99-100.

[22] Zhao XH, Fang RX, Rong J. Experiment study on comprehensive evaluation method of driving fatigue based on physiological signals. Journal of Beijing University of Technology. 2011;37(10):1511-1516.

[23] Guo WL, Gu J. A real-time ECG analysis algorithm for mobile ECG tele-monitoring system. Computer Simulation. 2014;31(9):272-276.

[24] Ji L, Wang H, Zhang C. Research on driver EEG characteristic and arm steering behavior. Chinese Journal of Scientific Instrument. 2015;36(9):2050-2056. 